\title{
Innovative video capsule endoscopy for detection of ubiquitously elongated small intestinal villi in Cronkhite-Canada syndrome
}

\author{
Hauke Sebastian Heinzow ${ }^{1}$, Wolfram Domschke ${ }^{1}$, Tobias Meister ${ }^{2}$ \\ ${ }^{1}$ Department of Medicine B, University Hospital Muenster, Germany \\ ${ }^{2}$ Helios Albert-Schweitzer University Teaching Hospital, Northeim, Germany
}

Videosurgery Miniinv 2014; 9 (1): 121-123

DOI: 10.5114/wiitm.2011.35782

\begin{abstract}
Cronkhite-Canada syndrome (CCS) is a rare non-familial disorder with multiple gastrointestinal polyps and ectodermal changes. Adenomatous and carcinomatous changes have been reported. Video capsule endoscopy is a useful non-invasive tool to reveal polypoid lesions of the gastrointestinal tract suspicious for malignancy. We report a case of a patient with CCS with excessively elongated intestinal villi resembling dense sea grass under water as well as multiple polyps of the intestinal mucosa revealed by video capsule endoscopy. This report presents for the first time small bowel video sequences of CCS qualifying video capsule endoscopy for screening purposes and early detection of malignancy.
\end{abstract}

Key words: Cronkhite-Canada syndrome, video capsule endoscopy, elongated intestinal villi.

\section{Introduction}

Video capsule endoscopy (VCE) as a new non-invasive diagnostic tool examining the small bowel has demonstrated its power in various studies [1-3]. There is growing acceptance for usage of VCE not only for obscure gastrointestinal bleeding but also to reveal suspicious polypoid lesions in polyposis syndromes and/or aphthoid/ulcerative lesions in patients with inflammatory bowel disease [4]. Cronkhite-Canada syndrome (CCS) is a rare non-familial disorder with multiple gastrointestinal polyps and ectodermal changes. Adenomatous and carcinomatous changes have been reported to occur in patients with CCS. Polyposis in the small bowel has been mainly confirmed by radiological findings and only in a few endoscopic studies [5]. For the first time we present high quality small bowel video sequences of CCS by video capsule endoscopy as a minimally invasive technique.

\section{Case report}

A 59-year-old female patient was admitted to our department with severe watery diarrhea, exsiccosis and shift in electrolyte balance. There was no significant family history. At admission the patient had a chronically ill appearance with alopecia and ectodermal changes in the form of onychodystrophy of finger- and toenails and discreet leg edema. Abdominal examination was normal. Initial laboratory tests showed anemia, hypoalbuminemia, hypoproteinemia, proteinuria, reduced serum calcium and serum chloride levels with elevated phosphate concentration. Other laboratory values were within the normal range, including tumor markers and parathormone. Colonoscopy revealed massive polyposis of the colon with subtotal stenosis leading to colectomy. Upper gastrointestinal (GI) endoscopy showed multiple polyps up to $4 \mathrm{~cm}$ in diameter of the gastric and duode- 


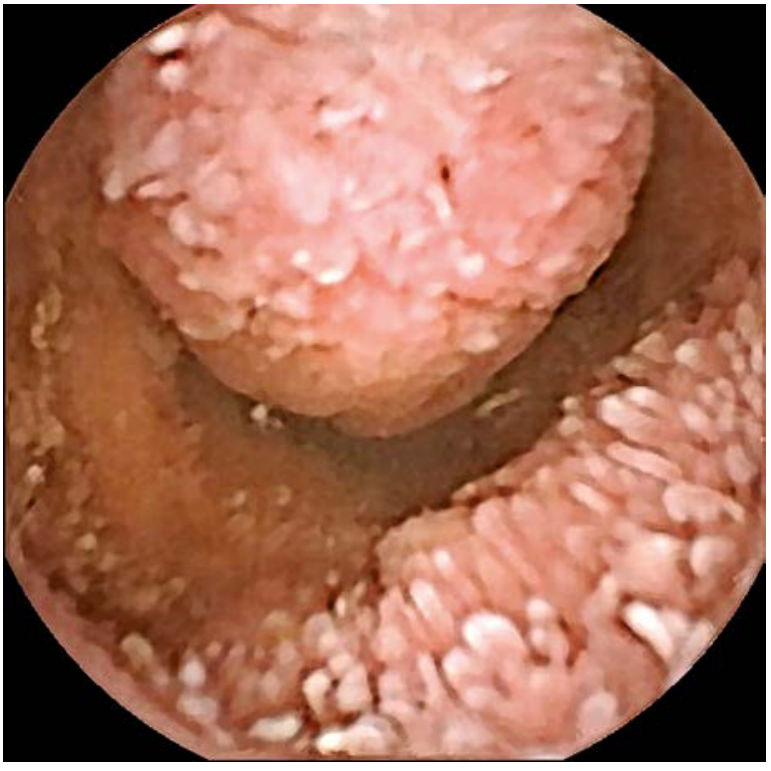

Photo 1. Video capsule endoscopic picture of the duodenum showing a typical polypoid lesion and elongated intestinal villi

nal mucosa, while the esophagus appeared normal (Photo 1). Histology demonstrated hamartomatous polyps of the duodenum (Photo 2). Immunohistochemistry confirmed the infiltration of the stroma by plasma cells as typically seen in CCS polyps (Photo 3). Further investigation by video capsule endoscopy (VCE) (PillCam SB, Given Imaging, Ltd., Yoqneam, Israel) confirmed excessively elongated intestinal villi resembling dense sea grass under water as well as multiple polyps with strawberry-red like central spots

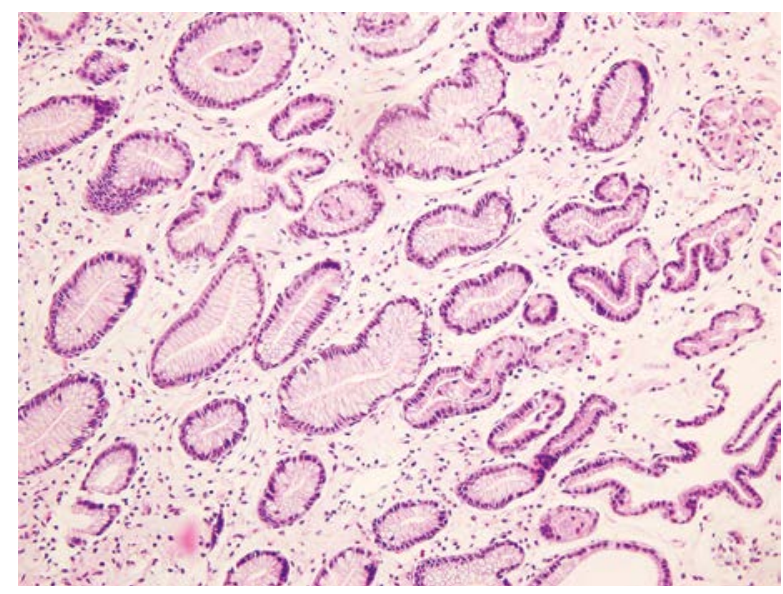

Photo 2. Histology $(H+E$ stained section) of a duodenal juvenile polyp with cystically enlarged glands filled with mucus of the intestinal mucosa. The endoscopic and histological findings as well as the clinical symptoms were typical of CCS. The exudative enteropathy due to the massive polyposis and status after colectomy had caused malabsorption and dehydration of the patient, who was treated by intravenous protein, caloric and electrolyte replacement, which led to a progressive improvement in general condition. Later on an intravenous port was implanted to guarantee sufficient additional parenteral nutritive substitution at home.

\section{Discussion}

The CCS belongs to a rare group of non-hereditary intestinal polyposis syndromes first described by Cronkhite and Canada in 1955 [6]. The etiology of CCS is still unknown. In CCS multiple juvenile polyps occur in the GI tract associated with abnormalities of skin and nails [7]. The symptom complex includes watery diarrhea with protein losing enteropathy, weight loss, alopecia and onychodystrophy. The present case was clinically and histologically consistent with CCS. The CCS is also associated with a higher incidence of Gl tract carcinomas [8]. Recent reports have recommended periodic follow-up by conventional endoscopy or conventional radiographic examinations [9]. For the first time, this report additionally presents video capsule sequences of CCS with description of elongated intestinal villi. Investigation of the small bowel has only recently become available by the invention of balloon enteroscopy and video

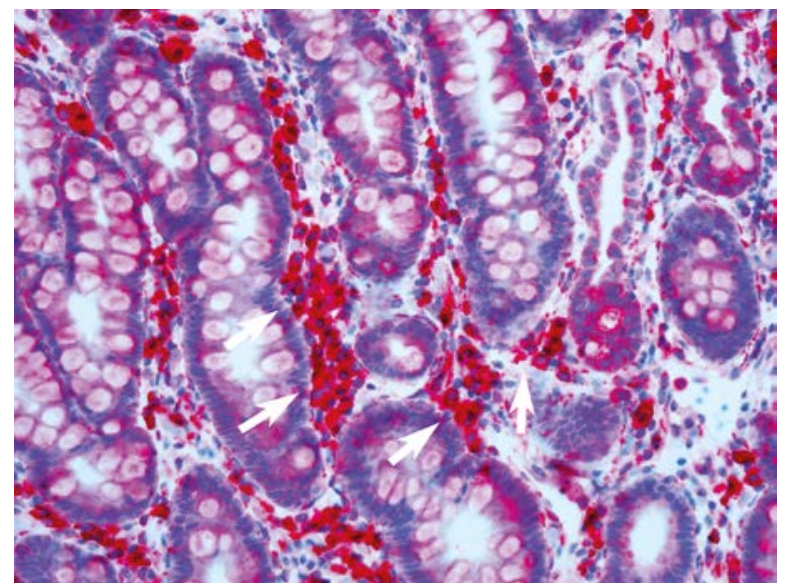

Photo 3. Immunohistochemistry with VS38cantibody reveals focally dense infiltration of the stroma by plasma cells (indicated by arrow) in hamartomatous polyps as typically seen in CCS 
capsule endoscopy. Thus, CCS patients should undergo a close follow-up by small bowel endoscopy due to an increased risk of the development of small bowel carcinomas. The authors consider video capsule endoscopy a useful tool for minimally invasive screening purposes. Suspicious lesions revealed by video capsule endoscopy should lead to performance of small bowel enteroscopy for biopsy or polypectomy and histological evaluation.

\section{References}

1. Hadithi M, Heine GD, Jacobs MA, et al. A prospective study comparing video capsule endoscopy with double-balloon enteroscopy in patients with obscure gastrointestinal bleeding. Am J Gastroenterol 2006; 101: 52-7.

2. Matsumoto T, Esaki M, Moriyama T, et al. Comparison of capsule endoscopy and enteroscopy with the double-balloon method in patients with obscure bleeding and polyposis. Endoscopy 2005 37: 827-32

3. Nakamura M, Niwa Y, Ohmiya N, et al. Preliminary comparison of capsule endoscopy and double-balloon enteroscopy in patients with suspected small-bowel bleeding. Endoscopy 2006; 38: 59-66.

4. Schulmann K, Hollerbach S, Kraus K, et al. Feasibility and diagnostic utility of video capsule endoscopy for the detection of small bowel polyps in patients with hereditary polyposis syndromes. Am J Gastroenterol 2005; 100: 27-37.

5. Koornstra J. Small bowel endoscopy in familial adenomatous polyposis and Lynch syndrome. Best Pract Res Clin Gastroenterol 2012; 26: 359-68.

6. Cronkhite LW Jr, Canada WJ. Generalized gastrointestinal polyposis; an unusual syndrome of polyposis, pigmentation, alopecia and onychotrophia. N Engl J Med 1955; 252: 1011-5.

7. Freeman K, Anthony PP, Miller DS, et al. Cronkhite Canada syndrome: a new hypothesis. Gut 1985; 26: 531-6.

8. Yashiro M, Kobayashi H, Kubo N, et al. Cronkhite-Canada syndrome containing colon cancer and serrated adenoma lesions. Digestion 2004; 69: 57-62.

9. Nagata K, Sato Y, Endo S, et al. CT endoscopy for the follow-up of Cronkhite-Canada syndrome. Int J Colorectal Dis 2007; 22: $1131-2$.

Received: 15.02.2013, accepted: 25.03.2013. 